\title{
Commentary to Gebel 2012: a quantitative review should apply meta-analytical methods
}

\author{
Peter Morfeld
}

Received: 28 February 2013/Accepted: 20 March 2013/Published online: 30 March 2013

(C) Springer-Verlag Berlin Heidelberg 2013

\begin{abstract}
Gebel (2012) performed a quantitative review of inhalation rat studies on the association of granular biopersistent dust exposures and lung cancer risk. The analytical methods applied are unreliable because they do not fulfil the requirements of current meta-analytical methods.
\end{abstract}

\section{Dear Editor,}

With interest, I read the quantitative review on the carcinogenic potency of GBP nano-materials and GPP micromaterials (Gebel 2012). GBP is used by the author as an abbreviation for "granular biopersistent particles." The author compared lung tumour rates in rat inhalation studies across different dose metrics to estimate an overall comparative potency factor for nano-GBP versus micro-GBP. Because this analysis may have impact on regulatory discussions I like to point at weaknesses of the chosen methodology. First, some of the metrics as given in Tables 4, 5 and 6 remained undefined. "Cumulative lung burden" cannot be derived from the data given in Table 1. No definition of this term was given. Second, the author performed a chain of calculations to gain summary statistics (e.g., he calculated ratios of averages of ratios). Statistical variability of the input data was ignored as if the

\section{P. Morfeld $(\square)$}

Institute for Occupational Epidemiology and Risk Assessment of Evonik Industries AG, Rellinghauser Straße 1-11, 45128 Essen, Germany

e-mail: peter.morfeld@evonik.com

\section{P. Morfeld}

Institute and Policlinic for Occupational Medicine, Environmental Medicine and Preventive Research, University of Cologne, Cologne, Germany data were true and known to the author with absolute certainty. Significance tests were performed on derived statistics as if the calculated numbers were single observations. The author measured the impact of different dose metrics on tumour rates with Pearson correlations as if they were regression coefficients. Thus, the chosen statistical procedures are inappropriate and may lead to unreliable findings (Rothman et al. 2008). Methods to perform quantitative reviews are well developed. Investigations that apply these methods are usually termed "meta-analyses" (e.g., Sutton et al. 2000). Fixed or random effect meta-analyses and meta-regressions are the usual approaches to combine findings from different (experimental) studies, to measure heterogeneity between these studies and to estimate potential interactions of the response with study variables. These methods are available in standard statistical software (e.g., Sterne et al. 2009). Without re-running the calculations of Gebel (2012) in terms of a meta-analysis, the derived quantitative summary statistics should not be mistaken as reliable input to regulatory discussions.

\section{References}

Gebel T (2012) Small difference in carcinogenic potency between GBP nanomaterials and GBP micromaterials. Arch Toxicol 86(7):995-1007

Rothman KJ, Greenland S, Lash TL (2008) Modern epidemiology, 3rd edn. Lippincott Williams \& Wilkins, Philadelphia

Sterne JAC, Newton HJ, Cox NJ (2009) Meta-analysis in stata: an updated collection from the stata journal. Stata Press, Texas

Sutton AJ, Abrams KR, Jones DR, Sheldon TA, Son F (2000) Methods for meta-analysis in medical research. Wiley, West Sussex 